\title{
Finite-Time Combination-Combination Synchronization for Hyperchaotic Systems
}

\author{
Huini Lin, ${ }^{1}$ Jianping Cai, ${ }^{1}$ and Jincheng Wang ${ }^{2}$ \\ ${ }^{1}$ Department of Mathematics, Zhangzhou Normal University, Zhangzhou 363000, China \\ ${ }^{2}$ College of Finance, Fujian Jiangxia University, Fuzhou 350108, China \\ Correspondence should be addressed to Huini Lin; 839480327@qq.com
}

Received 27 April 2013; Revised 13 July 2013; Accepted 24 July 2013

Academic Editor: Uchechukwu E. Vincent

Copyright (c) 2013 Huini Lin et al. This is an open access article distributed under the Creative Commons Attribution License, which permits unrestricted use, distribution, and reproduction in any medium, provided the original work is properly cited.

\begin{abstract}
A new type of finite-time synchronization with two drive systems and two response systems is presented. Based on the finitetime stability theory, step-by-step control and nonlinear control method, a suitable controller is designed to achieve finite-time combination-combination synchronization among four hyperchaotic systems. Numerical simulations are shown to verify the feasibility and effectiveness of the proposed control technique.
\end{abstract}

\section{Introduction}

As a new subject in 1980s, chaos almost covers all the fields of science. It is known that chaos is an interesting nonlinear phenomenon which may lead to irregularity and unpredictability in the dynamic system, and it has been intensively studied in the last three decades. Since Pecora and Carroll proposed the PC method to synchronize two chaotic systems in 1990 [1, 2], the study of synchronization of chaotic systems has been widely investigated due to their potential applications in various fields, for instance, in chemical reactions, biological systems, and secure communication. Over the past decades, a variety of control approaches such as adaptive control [3], linear feedback control [4], active control [5], and backstepping control [6] have been proposed for various types of synchronization, which include complete synchronization [7], projective synchronization [8, 9], general synchronization [10], lag synchronization [11], and novel compound synchronization [12].

Most of the aforementioned works are based on the synchronization scheme which consists of one drive system and one response system and can be seen as one-to-one system. However, we found it not secure and flexible enough in many real world applications, for instance, in secure communication. Recently, Runzi et al. presented a new type of synchronization with two drive systems and one response system [13]. Then, Sun et al. extended multi-to-one system to multi-to-two systems and reported a new type of synchronization, namely, combination-combination synchronization, where synchronization is achieved between two drive systems and two response systems [14]. The type of synchronization can improve the security of communication; for instance, we can split the transmitted signals into several parts, then load each part in different drive systems, and then restore it to the original signals by combining the received signals of different response systems correctly.

Notice that the mentioned literatures mainly investigated the asymptotic synchronization of chaotic systems. However, in the view of practical application, optimizing the synchronization time is more important than achieving synchronization asymptotically [15-19]. Recently, based on the stepby-step control method, Wang et al. realized the finite-time synchronization of two chaotic systems by designing a proper controller [15]. The method has the ability to achieve global stability in finite time. In addition, the step-by-step technique has the advantage of reducing controller complexity.

Motivated by the previous discussion, this paper aims to study the finite-time synchronization between a combination of two drive systems and a combination of two response systems in drive-response synchronization scheme. We have applied the finite-time stability theory to our analysis to achieve finite-time combination-combination synchronization. The step-by-step control method and nonlinear control technique are adopted to synchronize four different 
hyperchaotic systems. Numerical simulations are presented to verify the theoretical findings.

\section{The Finite-Time Combination-Combination Synchronization Scheme}

Consider the drive systems and response systems as follows:

$$
\begin{aligned}
\text { drive system 1 } & \dot{\mathbf{x}}_{1}=f_{1}\left(\mathbf{x}_{1}\right), \\
\text { drive system 2 } & \dot{\mathbf{x}}_{2}=f_{2}\left(\mathbf{x}_{2}\right), \\
\text { response system 1 } & \dot{\mathbf{y}}_{\mathbf{1}}=g_{1}\left(\mathbf{y}_{1}\right)+\mathbf{u}, \\
\text { response system 2 } & \dot{\mathbf{y}}_{2}=g_{2}\left(\mathbf{y}_{2}\right)+\mathbf{u}^{*},
\end{aligned}
$$

where $\mathbf{x}_{1}=\left(x_{1}, x_{2}, \ldots, x_{n}\right)^{T}, \mathbf{x}_{2}=\left(y_{1}, y_{2}, \ldots, y_{n}\right)^{T}, \mathbf{y}_{1}=\left(z_{1}\right.$, $\left.z_{2}, \ldots, z_{n}\right)^{T}$, and $\mathbf{y}_{2}=\left(w_{1}, w_{2}, \ldots, w_{n}\right)^{T}$ are the state vectors of systems (1), (2), (3), and (4), respectively. $f_{1}, f_{2}, g_{1}, g_{2}$ : $\mathbf{R}^{n} \rightarrow \mathbf{R}^{n}$ are four continuous functions, and $\mathbf{u}, \mathbf{u}^{*}: \mathbf{R}^{n} \times$ $\mathbf{R}^{n} \times \mathbf{R}^{n} \times \mathbf{R}^{n} \rightarrow \mathbf{R}^{n}$ are two controllers of the response systems (3) and (4) which will be designed, respectively.

Definition 1. If there exist four constant matrices $\mathbf{A}, \mathbf{B}, \mathbf{C}, \mathbf{D} \in$ $\mathbf{R}^{n}$ and $\mathbf{C} \neq \mathbf{0}, \mathbf{D} \neq \mathbf{0}$ such that $\lim _{t \rightarrow t} \| \mathbf{A} \mathbf{x}_{1}+\mathbf{B} \mathbf{x}_{\mathbf{2}}-\mathbf{C} \mathbf{y}_{\mathbf{1}}-$ $\mathbf{D} \mathbf{y}_{2} \|=0$ and $\left\|\mathbf{A} \mathbf{x}_{1}+\mathbf{B} \mathbf{x}_{2}-\mathbf{C y}_{\mathbf{1}}-\mathbf{D} \mathbf{y}_{2}\right\| \equiv 0$ for $t \geq t_{s}$, where $\mathbf{x}_{1}=\left(x_{1}, x_{2}, \ldots, x_{n}\right)^{T}, \mathbf{x}_{2}=\left(y_{1}, y_{2}, \ldots, y_{n}\right)^{T}, \mathbf{y}_{1}=\left(z_{1}\right.$, $\left.z_{2}, \ldots, z_{n}\right)^{T}$, and $\mathbf{y}_{2}=\left(w_{1}, w_{2}, \ldots, w_{n}\right)^{T}$, one gets that the drive systems (1) and (2) are realized as finite-time combination-combination synchronization with the response systems (3) and (4), where $\|\cdot\|$ represents the matrix norm.

Lemma 2 (see [15]). Assume that a continuous, positivedefinite function $V(t)$ satisfies the following differential inequality:

$$
\dot{V}(t) \leq-c V^{\eta}(t), \quad \forall t \geq t_{0}, V\left(t_{0}\right) \geq 0
$$

where $c>0$ and $0<\eta<1$ are constants. Then, for any initial time $t_{0}, V(t)$ satisfies

$$
\begin{gathered}
V^{1-\eta}(t) \leq V^{1-\eta}\left(t_{0}\right)-c(1-\eta)\left(t-t_{0}\right), \quad t_{0} \leq t \leq t_{1}, \\
V(t) \equiv 0, \quad \forall t \geq t_{1},
\end{gathered}
$$

with $t_{1}$ given by

$$
t_{1}=t_{0}+\frac{V^{1-\eta}\left(t_{0}\right)}{c(1-\eta)} .
$$

Thus, for any initial value $V\left(t_{0}\right)$, the system (5) has $V(t)=0$ in $t_{1}=t_{0}+V^{1-\eta}\left(t_{0}\right) / c(1-\eta)$; that is, the system can achieve global stability in finite time.

\section{Problem Statement and Control Scheme}

3.1. Problem Statement. Consider two identity hyperchaotic Chen systems as the drive systems [20]:

$$
\begin{gathered}
\dot{x}_{1}=a\left(y_{1}-x_{1}\right)+w_{1}, \\
\dot{y}_{1}=d x_{1}-x_{1} z_{1}+c y_{1}, \\
\dot{z}_{1}=x_{1} y_{1}-b z_{1}, \\
\dot{w}_{1}=y_{1} z_{1}+r w_{1}, \\
\dot{x}_{2}=a\left(y_{2}-x_{2}\right)+w_{2}, \\
\dot{y}_{2}=d x_{2}-x_{2} z_{2}+c y_{2}, \\
\dot{z}_{2}=x_{2} y_{2}-b z_{2}, \\
\dot{w}_{2}=y_{2} z_{2}+r w_{2} .
\end{gathered}
$$

Consider two identity hyperchaotic Lorenz systems as the response systems [21]:

$$
\begin{gathered}
\dot{x}_{3}=a_{1}\left(y_{3}-x_{3}\right)+w_{3}+u_{1}, \\
\dot{y}_{3}=c_{1} x_{3}-y_{3}-x_{3} z_{3}+u_{2}, \\
\dot{z}_{3}=x_{3} y_{3}-b_{1} z_{3}+u_{3}, \\
\dot{w}_{3}=-y_{3} z_{3}+r_{1} w_{3}+u_{4}, \\
\dot{x}_{4}=a_{1}\left(y_{4}-x_{4}\right)+w_{4}+u_{1}^{*}, \\
\dot{y}_{4}=c_{1} x_{4}-y_{4}-x_{4} z_{4}+u_{2}^{*}, \\
\dot{z}_{4}=x_{4} y_{4}-b_{1} z_{4}+u_{3}^{*}, \\
\dot{w}_{4}=-y_{4} z_{4}+r_{1} w_{4}+u_{4}^{*},
\end{gathered}
$$

where $\mathbf{u}=\left(u_{1}, u_{2}, u_{3}, u_{4}\right)^{T}$ and $\mathbf{u}^{*}=\left(u_{1}^{*}, u_{2}^{*}, u_{3}^{*}, u_{4}^{*}\right)^{T}$ are two controllers of the response systems (10) and (11) which will be designed, respectively.

Without loss of generality, we choose $\mathbf{A}=\operatorname{diag}\left(\alpha_{1}, \alpha_{2}\right.$, $\left.\alpha_{3}, \alpha_{4}\right), \mathbf{B}=\operatorname{diag}\left(\beta_{1}, \beta_{2}, \beta_{3}, \beta_{4}\right), \mathbf{C}=\operatorname{diag}\left(\gamma_{1}, \gamma_{2}, \gamma_{3}, \gamma_{4}\right)$, and $\mathbf{D}=\operatorname{diag}(1,1,1,1)$.

The objective of the synchronization scheme is to design a suitable controller $\mathbf{U}(x, y, z, w)$ such that $\lim _{t \rightarrow t_{s}} \| \mathbf{A x}_{\mathbf{1}}+\mathbf{B x}_{\mathbf{2}}-$ $\mathbf{C y}_{1}-\mathbf{D} \mathbf{y}_{2} \|=0$ and $\left\|\mathbf{A} \mathbf{x}_{1}+\mathbf{B} \mathbf{x}_{2}-\mathbf{C y}_{1}-\mathbf{D} \mathbf{y}_{2}\right\| \equiv 0$ for $t \geq$ $t_{s}$, where $\mathbf{x}_{1}=\left(x_{1}, x_{2}, \ldots, x_{n}\right)^{T}, \mathbf{x}_{2}=\left(y_{1}, y_{2}, \ldots, y_{n}\right)^{T}, \mathbf{y}_{1}=$ $\left(z_{1}, z_{2}, \ldots, z_{n}\right)^{T}$, and $\mathbf{y}_{2}=\left(w_{1}, w_{2}, \ldots, w_{n}\right)^{T}$; that is, the drive systems (8) and (9) are realized as finite-time combinationcombination synchronization with the response systems (10) and (11).

3.2. The Control Scheme. Let $e_{1}=\alpha_{1} x_{1}+\beta_{1} x_{2}-\gamma_{1} x_{3}-x_{4}$, $e_{2}=\alpha_{2} y_{1}+\beta_{2} y_{2}-\gamma_{2} y_{3}-y_{4}, e_{3}=\alpha_{3} z_{1}+\beta_{3} z_{2}-\gamma_{3} z_{3}-z_{4}$, and $e_{4}=\alpha_{4} w_{1}+\beta_{4} w_{2}-\gamma_{4} w_{3}-w_{4}$. The controller to be designed is $U_{1}=\gamma_{1} u_{1}+u_{1}^{*}, U_{2}=\gamma_{2} u_{2}+u_{2}^{*}, U_{3}=\gamma_{3} u_{3}+u_{3}^{*}$, and $U_{4}=\gamma_{4} u_{4}+u_{4}^{*}$. 
Thus, we can get the error system as follows:

$$
\begin{aligned}
\dot{e}_{1}= & -a e_{1}+e_{4}+a\left(\alpha_{1} y_{1}+\beta_{1} y_{2}-\gamma_{1} x_{3}-x_{4}\right) \\
& -a_{1}\left(\gamma_{1} y_{3}-\gamma_{1} x_{3}+y_{4}-x_{4}\right)+\left(\alpha_{1}-\alpha_{4}\right) w_{1} \\
& +\left(\beta_{1}-\beta_{4}\right) w_{2}-\left(\gamma_{1}-\gamma_{4}\right) w_{3}-U_{1}, \\
\dot{e}_{2}= & c_{1} e_{1}+c e_{2}+(c+1)\left(\gamma_{2} y_{3}+y_{4}\right)+d\left(\alpha_{2} x_{1}+\beta_{2} x_{2}\right) \\
& -c_{1}\left[\alpha_{1} x_{1}+\beta_{1} x_{2}+\left(\gamma_{2}-\gamma_{1}\right) x_{3}\right] \\
& -\left(\alpha_{2} x_{1} z_{1}+\beta_{2} x_{2} z_{2}-\gamma_{2} x_{3} z_{3}-x_{4} z_{4}\right)-U_{2}, \\
\dot{e}_{3}= & -b e_{3}+\left(b_{1}-b\right)\left(\gamma_{3} z_{3}+z_{4}\right) \\
& +\left(\alpha_{3} x_{1} y_{1}+\beta_{3} x_{2} y_{2}-\gamma_{3} x_{3} y_{3}-x_{4} y_{4}\right)-U_{3}, \\
\dot{e}_{4}= & r e_{4}+\left(r-r_{1}\right)\left(\gamma_{4} w_{3}+w_{4}\right) \\
& +\left(\alpha_{4} y_{1} z_{1}+\beta_{4} y_{2} z_{2}+\gamma_{4} y_{3} z_{3}+y_{4} z_{4}\right)-U_{4} .
\end{aligned}
$$

Our aim is to design a suitable controller, such that the drive systems (8) and (9) are realized as combinationcombination synchronization with the response systems (10) and (11) in finite time. Then, the problem is changed to design a suitable controller, such that the error system (12) achieves the finite-time stability at the origin.

The design plan and its steps are as follows.

Step 1. Choose

$$
\begin{aligned}
U_{4}= & \left(r-r_{1}\right)\left(\gamma_{4} w_{3}+w_{4}\right) \\
& +\left(\alpha_{4} y_{1} z_{1}+\beta_{4} y_{2} z_{2}+\gamma_{4} y_{3} z_{3}+y_{4} z_{4}\right)+k_{4} e_{4}+e_{4}^{\alpha},
\end{aligned}
$$

where

$$
\begin{aligned}
u_{4}=\frac{1}{\gamma_{4}}\left[\left(r-r_{1}\right)\left(\gamma_{4} w_{3}+w_{4}\right)\right. & \\
& \left.+\left(\alpha_{4} y_{1} z_{1}+\beta_{4} y_{2} z_{2}+\gamma_{4} y_{3} z_{3}+y_{4} z_{4}\right)\right], \\
u_{4}^{*} & =k_{4} e_{4}+e_{4}^{\alpha}, \quad k_{4}>r, \alpha=\frac{q}{p}
\end{aligned}
$$

is a proper rational number, and $p$ is a positive odd number, $p>q$.

Substituting $U_{4}$ into the fourth equation of (12), we get

$$
\dot{e}_{4}=\left(r-k_{4}\right) e_{4}-e_{4}^{\alpha} .
$$

Choose a candidate Lyapunov function

$$
V_{4}=\frac{1}{2} e_{4}^{2}
$$

Thus, the derivative of $V_{4}$ along the solution of error equation (15) is

$$
\begin{aligned}
\dot{V}_{4} & =\left(r-k_{4}\right) e_{4}^{2}-e_{4}^{\alpha+1} \leq-e_{4}^{\alpha+1} \\
& =-2^{(\alpha+1) / 2}\left(\frac{1}{2} e_{4}^{2}\right)^{(\alpha+1) / 2}=-2^{(\alpha+1) / 2} V_{4}^{(\alpha+1) / 2} .
\end{aligned}
$$

According to Lemma 2, the system (15) is finite-time stability, which implies that there exists $T_{1}>0$, such that $e_{4} \equiv 0$ for $t \geq T_{1}$.

Step 2. Choose

$$
\begin{aligned}
U_{1}= & a\left(\alpha_{1} y_{1}+\beta_{1} y_{2}-\gamma_{1} x_{3}-x_{4}\right) \\
& -a_{1}\left(\gamma_{1} y_{3}-\gamma_{1} x_{3}+y_{4}-x_{4}\right) \\
& +\left(\alpha_{1}-\alpha_{4}\right) w_{1}+\left(\beta_{1}-\beta_{4}\right) w_{2}-\left(\gamma_{1}-\gamma_{4}\right) w_{3} \\
& +k_{1} e_{1}+e_{1}^{\beta},
\end{aligned}
$$

where

$$
\begin{aligned}
u_{1}=\frac{1}{\gamma_{1}}[ & a\left(\alpha_{1} y_{1}+\beta_{1} y_{2}-\gamma_{1} x_{3}-x_{4}\right) \\
& -a_{1}\left(\gamma_{1} y_{3}-\gamma_{1} x_{3}+y_{4}-x_{4}\right) \\
& +\left(\alpha_{1}-\alpha_{4}\right) w_{1}+\left(\beta_{1}-\beta_{4}\right) w_{2} \\
& \left.-\left(\gamma_{1}-\gamma_{4}\right) w_{3}\right], \\
u_{1}^{*}=k_{1} e_{1} & +e_{1}^{\beta}, \quad k_{1}>-a, 0<\beta<1 .
\end{aligned}
$$

For $t>T_{1}$, substituting $U_{1}$ into the first equation of (12), we get

$$
\dot{e}_{1}=-\left(k_{1}+a\right) e_{1}-e_{1}^{\beta} .
$$

Choose a candidate Lyapunov function

$$
V_{1}=\frac{1}{2} e_{1}^{2}
$$

Thus, the derivative of $V_{1}$ along the solution of error equation (20) is

$$
\begin{aligned}
\dot{V}_{1} & =-\left(k_{1}+a\right) e_{1}^{2}-e_{1}^{\beta+1} \leq-e_{1}^{\beta+1} \\
& =-2^{(\beta+1) / 2}\left(\frac{1}{2} e_{1}^{2}\right)^{(\beta+1) / 2}=-2^{(\beta+1) / 2} V_{1}^{(\beta+1) / 2} .
\end{aligned}
$$

According to Lemma 2, the system (20) is finite-time stability, which implies that there exists $T_{2}>0$, such that $e_{1} \equiv 0$ for $t \geq T_{2}$.

Step 3. Choose

$$
\begin{aligned}
U_{2}= & (c+1)\left(\gamma_{2} y_{3}+y_{4}\right)+d\left(\alpha_{2} x_{1}+\beta_{2} x_{2}\right) \\
& -c_{1}\left[\alpha_{1} x_{1}+\beta_{1} x_{2}+\left(\gamma_{2}-\gamma_{1}\right) x_{3}\right] \\
& -\left(\alpha_{2} x_{1} z_{1}+\beta_{2} x_{2} z_{2}-\gamma_{2} x_{3} z_{3}-x_{4} z_{4}\right) \\
& +k_{2} e_{2}+e_{2}^{\gamma},
\end{aligned}
$$

where

$$
\begin{aligned}
u_{2}=\frac{1}{\gamma_{2}}\left[(c+1)\left(\gamma_{2} y_{3}+y_{4}\right)+d\left(\alpha_{2} x_{1}+\beta_{2} x_{2}\right)\right. \\
\quad-c_{1}\left[\alpha_{1} x_{1}+\beta_{1} x_{2}+\left(\gamma_{2}-\gamma_{1}\right) x_{3}\right] \\
\left.\quad-\left(\alpha_{2} x_{1} z_{1}+\beta_{2} x_{2} z_{2}-\gamma_{2} x_{3} z_{3}-x_{4} z_{4}\right)\right] \\
u_{2}^{*}=k_{2} e_{2}+e_{2}^{\gamma}, \quad k_{2}>c, 0<\gamma<1 .
\end{aligned}
$$




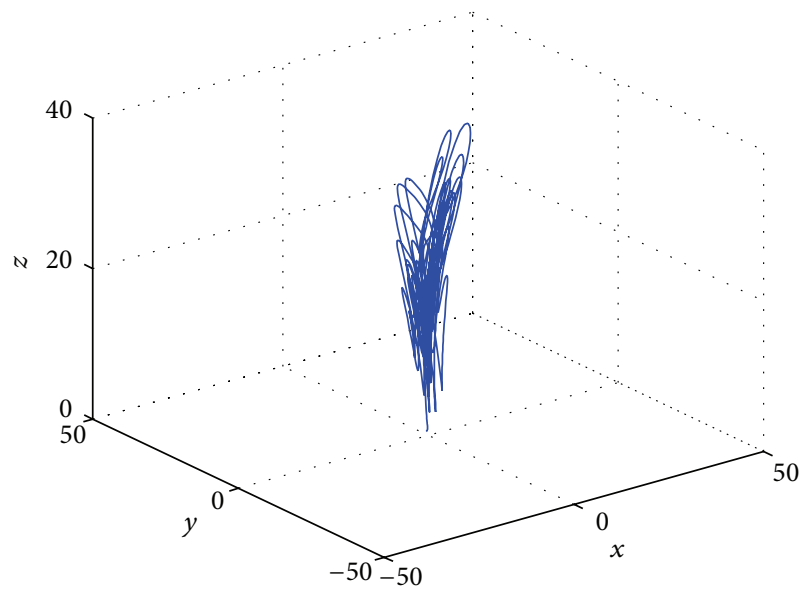

(a)

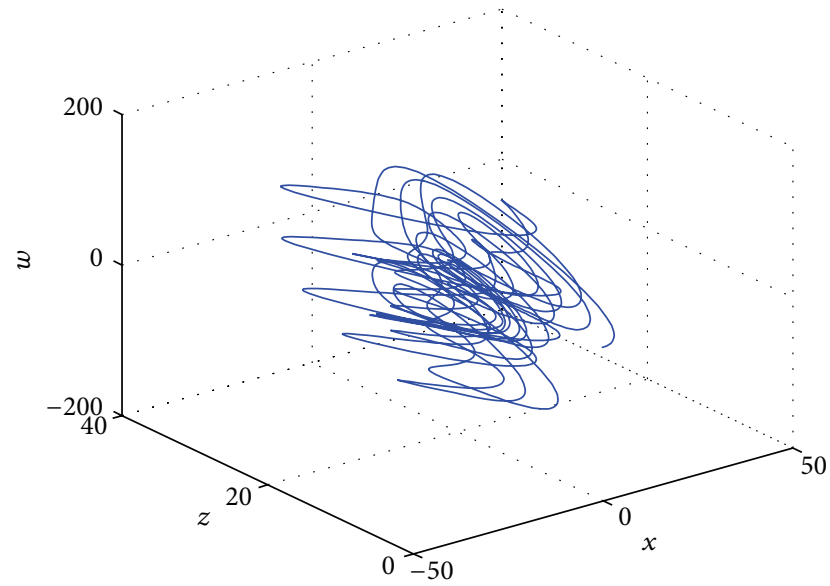

(c)

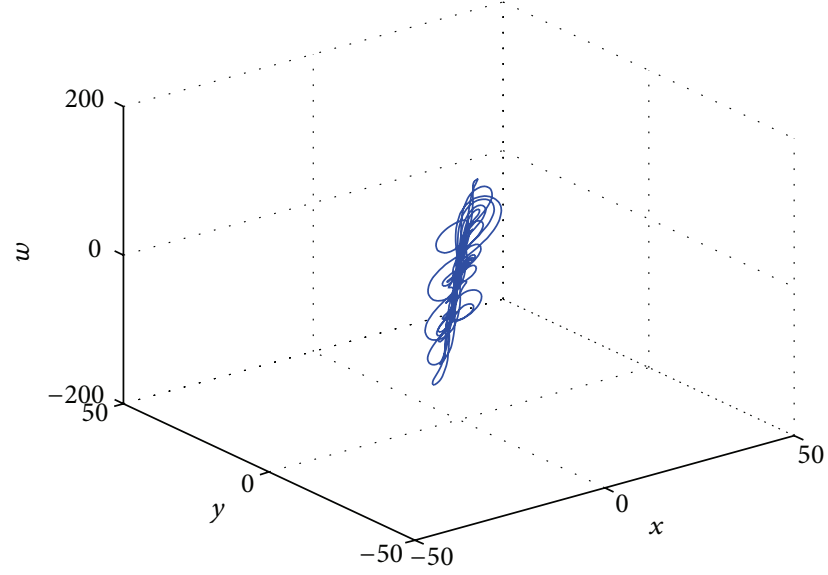

(b)

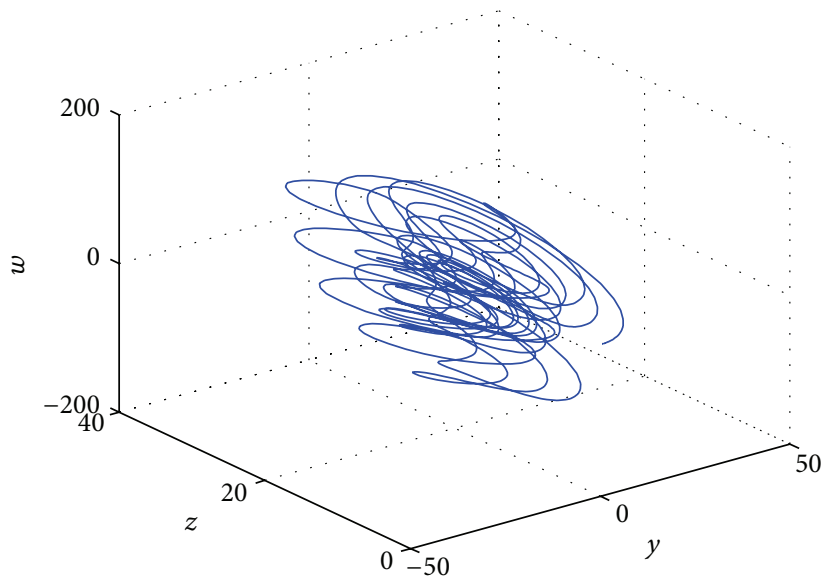

(d)

FIgURE 1: Hyperchaotic attractors of the hyperchaotic Chen system with $a=35, b=3, c=12, d=7$, and $r=0.5$.

For $t>T_{2}$, substituting $U_{2}$ into the second equation of (12), we get

$$
\dot{e}_{2}=\left(-k_{2}+c\right) e_{2}-e_{2}^{\gamma}
$$

Choose a candidate Lyapunov function

$$
V_{2}=\frac{1}{2} e_{2}^{2}
$$

Thus, the derivative of $V_{2}$ along the solution of error equation (25) is

$$
\begin{aligned}
\dot{V}_{2} & =\left(-k_{2}+c\right) e_{2}^{2}-e_{2}^{\gamma+1} \leq-e_{2}^{\gamma+1} \\
& =-2^{(\gamma+1) / 2}\left(\frac{1}{2} e_{2}^{2}\right)^{(\gamma+1) / 2}=-2^{(\gamma+1) / 2} V_{2}^{(\gamma+1) / 2} .
\end{aligned}
$$

According to Lemma 2, the system (25) is finite-time stability, which implies that there exists $T_{3}>0$, such that $e_{2} \equiv 0$ for $t \geq T_{3}$.
Step 4. Choose

$$
\begin{aligned}
U_{3}= & \left(b_{1}-b\right)\left(\gamma_{3} z_{3}+z_{4}\right) \\
& +\left(\alpha_{3} x_{1} y_{1}+\beta_{3} x_{2} y_{2}-\gamma_{3} x_{3} y_{3}-x_{4} y_{4}\right) \\
& +k_{3} e_{3}+e_{3}^{\delta},
\end{aligned}
$$

where

$$
\begin{aligned}
u_{3}=\frac{1}{\gamma_{3}}[ & \left(b_{1}-b\right)\left(\gamma_{3} z_{3}+z_{4}\right) \\
& \left.+\left(\alpha_{3} x_{1} y_{1}+\beta_{3} x_{2} y_{2}-\gamma_{3} x_{3} y_{3}-x_{4} y_{4}\right)\right], \\
u_{3}^{*}= & k_{3} e_{3}+e_{3}^{\delta}, \quad k_{3}>-b, 0<\delta<1 .
\end{aligned}
$$

For $t>T_{3}$, substituting $U_{3}$ into the third equation of (12), we get

$$
\dot{e}_{3}=-\left(b+k_{3}\right) e_{3}-e_{3}^{\delta}
$$




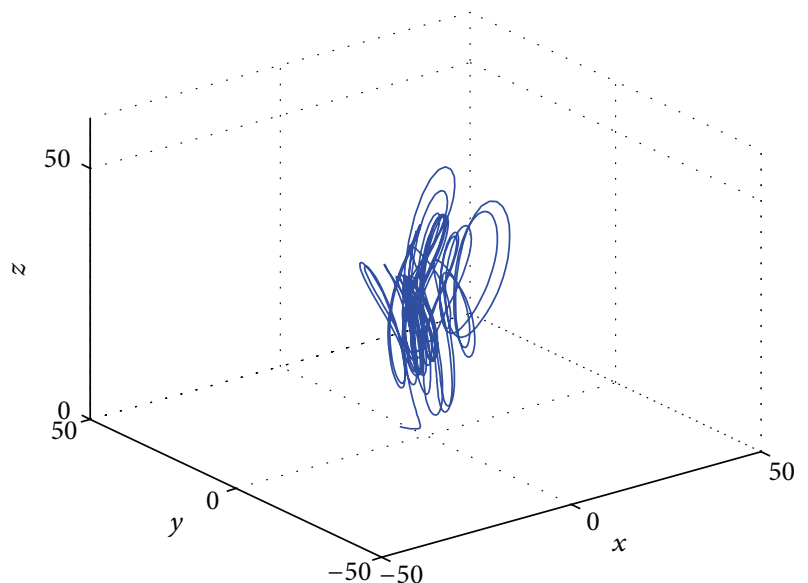

(a)

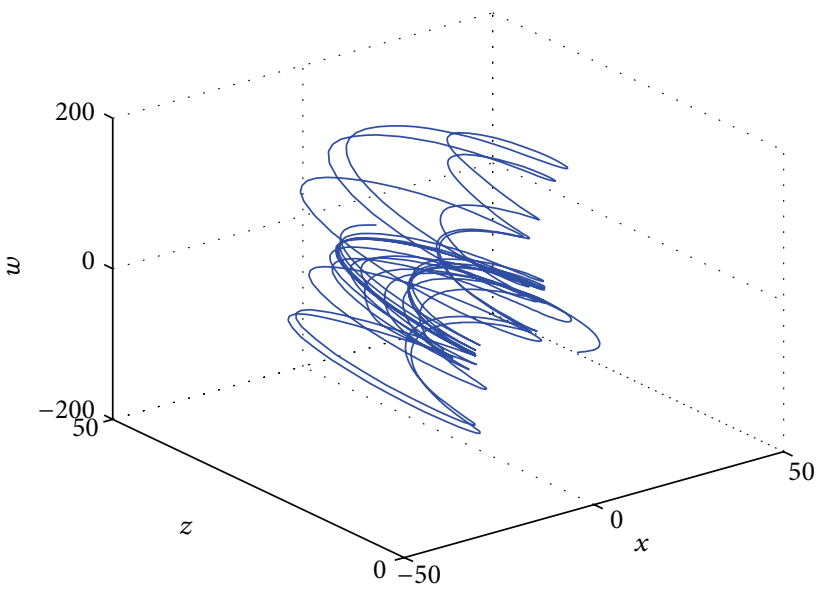

(c)

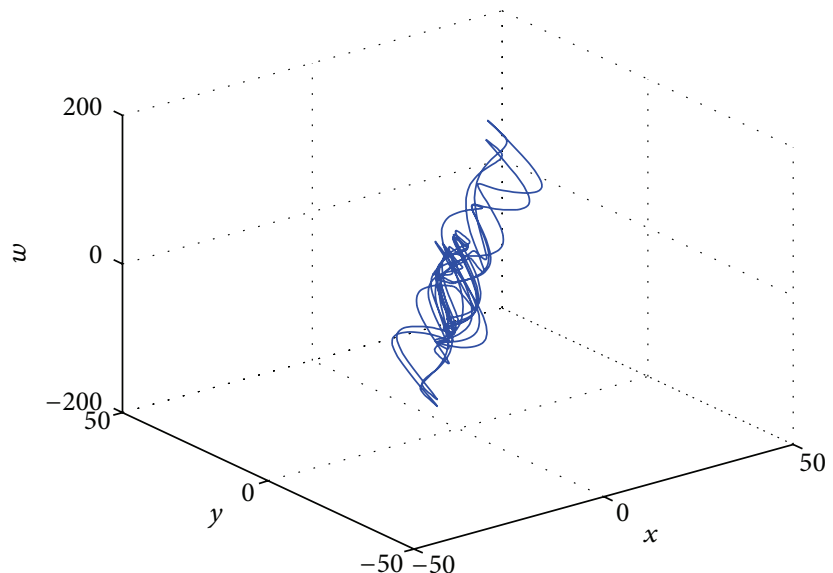

(b)

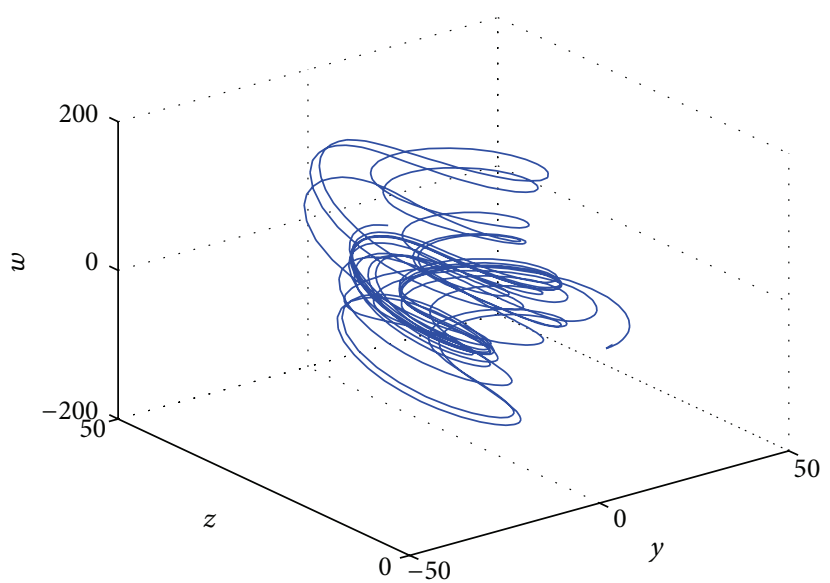

(d)

Figure 2: Hyperchaotic attractors of the hyperchaotic Lorenz system with $a_{1}=10, b_{1}=8 / 3, c_{1}=28$, and $r_{1}=-1$.

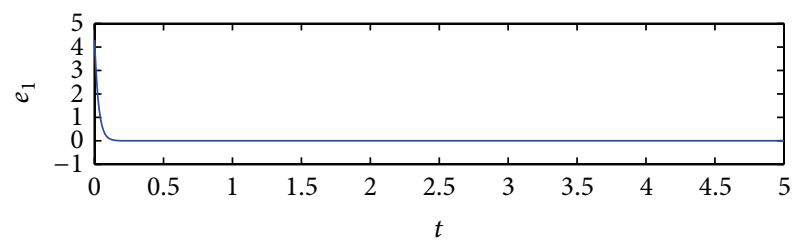

(a)

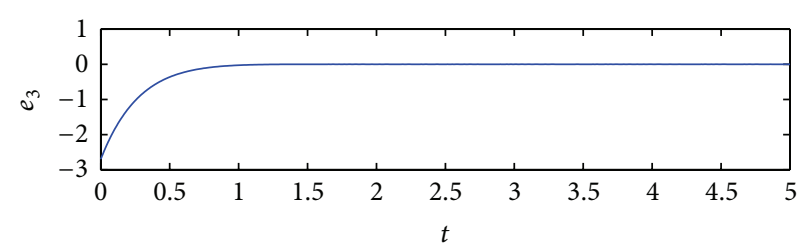

(c)

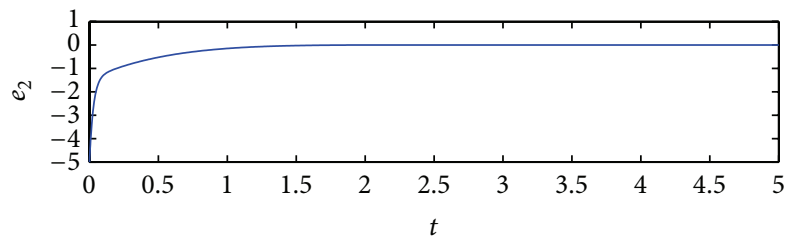

(b)

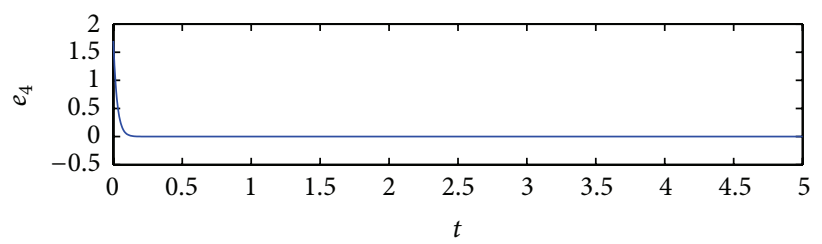

(d)

FIGURE 3: The errors between drive systems (8) and (9) and response systems (10) and (11). 
Choose a candidate Lyapunov function

$$
V_{3}=\frac{1}{2} e_{3}^{2}
$$

Thus, the derivative of $V_{3}$ along the solution of error equation (30) is

$$
\begin{aligned}
\dot{V}_{3} & =-\left(b+k_{3}\right) e_{3}^{2}-e_{3}^{\delta+1} \leq-e_{3}^{\delta+1} \\
& =-2^{(\delta+1) / 2}\left(\frac{1}{2} e_{3}^{2}\right)^{(\delta+1) / 2}=-2^{(\delta+1) / 2} V_{3}^{(\delta+1) / 2} .
\end{aligned}
$$

According to Lemma 2, the system (30) is finite-time stability, which implies that there exists $T_{4}>0$, such that $e_{3} \equiv 0$ for $t \geq T_{4}$.

The controller is designed as follows:

$$
\begin{aligned}
U_{1}= & a\left(\alpha_{1} y_{1}+\beta_{1} y_{2}-\gamma_{1} x_{3}-x_{4}\right) \\
& -a_{1}\left(\gamma_{1} y_{3}-\gamma_{1} x_{3}+y_{4}-x_{4}\right) \\
& +\left(\alpha_{1}-\alpha_{4}\right) w_{1}+\left(\beta_{1}-\beta_{4}\right) w_{2}-\left(\gamma_{1}-\gamma_{4}\right) w_{3} \\
& +k_{1} e_{1}+e_{1}^{\beta}, \\
U_{2}= & (c+1)\left(\gamma_{2} y_{3}+y_{4}\right)+d\left(\alpha_{2} x_{1}+\beta_{2} x_{2}\right) \\
& -c_{1}\left[\alpha_{1} x_{1}+\beta_{1} x_{2}+\left(\gamma_{2}-\gamma_{1}\right) x_{3}\right] \\
& -\left(\alpha_{2} x_{1} z_{1}+\beta_{2} x_{2} z_{2}-\gamma_{2} x_{3} z_{3}-x_{4} z_{4}\right) \\
& +k_{2} e_{2}+e_{2}^{\gamma}, \\
U_{3}= & \left(b_{1}-b\right)\left(\gamma_{3} z_{3}+z_{4}\right) \\
& +\left(\alpha_{3} x_{1} y_{1}+\beta_{3} x_{2} y_{2}-\gamma_{3} x_{3} y_{3}-x_{4} y_{4}\right)+k_{3} e_{3}+e_{3}^{\delta}, \\
U_{4}= & \left(r-r_{1}\right)\left(\gamma_{4} w_{3}+w_{4}\right) \\
& +\left(\alpha_{4} y_{1} z_{1}+\beta_{4} y_{2} z_{2}+\gamma_{4} y_{3} z_{3}+y_{4} z_{4}\right)+k_{4} e_{4}+e_{4}^{\alpha} .
\end{aligned}
$$

According to what we discussed previously, we can obtain this conclusion that the error system (12) achieves finite-time stability under the control of the controller (33). Furthermore, the drive systems (8) and (9) are realized as combinationcombination synchronization with the response systems (10) and (11) in finite time $T \leq T_{s}$, where $T_{s}=T_{1}+T_{2}+T_{3}+T_{4}$.

\section{Numerical Simulation}

To verify the effectiveness of the proposed finite-time synchronization method, we consider the hyperchaotic Chen system with the parameters $a=35, b=3, c=12, d=7$, and $r=0.5$. The hyperchaotic attractor of the system is shown in Figure 1.

Consider the hyperchaotic Lorenz system with the parameters $a_{1}=10, b_{1}=8 / 3, c_{1}=28$, and $r_{1}=-1$. The hyperchaotic attractor of the system is shown in Figure 2.
In the following simulation, we assume $\alpha_{1}=\alpha_{2}=\alpha_{3}=$ $\alpha_{4}=1, \beta_{1}=\beta_{2}=\beta_{3}=\beta_{4}=1$, and $\gamma_{1}=\gamma_{2}=\gamma_{3}=$ $\gamma_{4}=1$, and the initial states for the drive systems and response systems are arbitrarily given by $\left(x_{1}(0)\right.$, $\left.y_{1}(0), z_{1}(0), w_{1}(0)\right)^{T}=(0.1,0.5,1,0.2)^{T},\left(x_{2}(0), y_{2}(0), z_{2}(0)\right.$, $\left.w_{2}(0)\right)^{T}=(0.2,1,0.3,2)^{T},\left(x_{3}(0), y_{3}(0), z_{3}(0), w_{3}(0)\right)^{T}=$ $(-1,1.5,3,-2)^{T}$, and $\left(x_{4}(0), y_{4}(0), z_{4}(0), w_{4}(0)\right)^{T}=(-3,5$, $1,2.5)^{T}$. Then, and we choose the synchronization controller with $k_{1}=0, k_{2}=13, k_{3}=0, k_{4}=36, \alpha=\beta=\gamma=\delta=2 / 3$. The synchronization evolution for this controller is shown in Figure 3.

The error vector is achieved to zero which implies that systems (8), (9) and (10), (11) have achieved finite-time combination-combination synchronization.

\section{Conclusion}

In this paper, the problem of finite-time combinationcombination synchronization with two drive systems and two response systems was investigated. Based on the finitetime stability theory, the step-by-step control and nonlinear control approach, a suitable controller was introduced. The simulation results demonstrated that the proposed controller works well for synchronizing four hyperchaotic systems in finite time.

\section{Acknowledgments}

The authors would like to thank the Editor and the referees for their valuable comments and suggestions that helped to improve the quality of this paper. This work was supported by the National Natural Science Foundation of China (Grant no. 61074012) and the Natural Science Foundation of Fujian Province (Grant no. 2011J01025).

\section{References}

[1] L. M. Pecora and T. L. Carroll, "Synchronization in chaotic systems," Physical Review Letters, vol. 64, no. 8, pp. 821-824, 1990.

[2] L. M. Pecora, T. L. Carroll, G. A. Johnson, D. J. Mar, and J. F. Heagy, "Fundamentals of synchronization in chaotic systems, concepts, and applications," Chaos, vol. 7, no. 4, pp. 520-543, 1997.

[3] T. L. Liao and S. H. Tsai, "Adaptive synchronization of chaotic systems and its application to secure communications," Chaos, solitons and fractals, vol. 11, no. 9, pp. 1387-1396, 2000.

[4] M. T. Yassen, "Controlling chaos and synchronization for new chaotic system using linear feedback control," Chaos, Solitons and Fractals, vol. 26, no. 3, pp. 913-920, 2005.

[5] Y. Lei, W. Xu, and H. Zheng, "Synchronization of two chaotic nonlinear gyros using active control," Physics Letters A, vol. 343, no. 1-3, pp. 153-158, 2005.

[6] S. Tong, C. Li, and Y. Li, "Fuzzy adaptive observer backstepping control for MIMO nonlinear systems," Fuzzy Sets and Systems, vol. 160, no. 19, pp. 2755-2775, 2009.

[7] C. Yao, Q. Zhao, and J. Yu, "Complete synchronization induced by disorder in coupled chaotic lattices," Physics Letters A, vol. 377, no. 5, pp. 370-377, 2013. 
[8] J. Sun, Y. Shen, G. Zhang et al., "General hybrid projective complete dislocated synchronization with non-derivative and derivative coupling based on parameter identification in several chaotic and hyperchaotic systems," Chinese Physics B, vol. 22, no. 4, pp. 040508-040518, 2013.

[9] J. Sun, Y. Shen, and G. Zhang, "Transmission projective synchronization of multi-systems with non-delayed and delayed coupling via impulsive control," Chaos, vol. 22, no. 4, pp. 043107-043116, 2012.

[10] H. G. Enjieu Kadji and R. Yamapi, "General synchronization dynamics of coupled Van der Pol-Duffing oscillators," Physica A, vol. 370, no. 2, pp. 316-328, 2006.

[11] Y. Shen and J. Wang, "Robustness analysis of global exponential stability of recurrent neural networks in the presence of time delays and random disturbances," IEEE Transactions on Neural Networks and Learning Systems, vol. 23, no. 1, pp. 87-96, 2012.

[12] J. Sun, Y. Shen, Q. Yin et al., "Compound synchronization of four memristor chaotic oscillator systems and secure communication," Chaos, vol. 23, no. 1, pp. 013140-013149, 2013.

[13] R. Luo, Y. Wang, and S. Deng, "Combination synchronization of three classic chaotic systems using active backstepping design," Chaos, vol. 21, no. 4, pp. 043114-043119, 2011.

[14] J. Sun, Y. Shen, G. Zhang et al., "Combination-combination synchronization among four identical or different chaotic systems," Nonlinear Dynamics, vol. 73, no. 3, pp. 1211-1222, 2013.

[15] H. Wang, Z. Han, Q. Xie, and W. Zhang, "Finite-time chaos synchronization of unified chaotic system with uncertain parameters," Communications in Nonlinear Science and Numerical Simulation, vol. 14, no. 5, pp. 2239-2247, 2009.

[16] Y. Yang and X. Wu, "Global finite-time synchronization of a class of the non-autonomous chaotic systems," Nonlinear Dynamics, vol. 70, no. 1, pp. 197-208, 2012.

[17] U. E. Vincent and R. Guo, "Finite-time synchronization for a class of chaotic and hyperchaotic systems via adaptive feedback controller," Physics Letters A, vol. 375, no. 24, pp. 2322-2326, 2011.

[18] R. Luo and Y. Wang, "Finite-time stochastic combination synchronization of three different chaotic systems and its application in secure communication," Chaos, vol. 22, no. 2, pp. 023109-023118, 2012.

[19] P. He, S. Ma, and T. Fan, "Finite-time mixed outer synchronization of complex networks with coupling time-varying delay," Chaos, vol. 22, no. 4, pp. 043151-043161, 2012.

[20] Y. X. Li, W. K. S. Tang, and G. R. Chen, "Generating hyperchaos via state feedback control," International Journal of Bifurcation and Chaos in Applied Sciences and Engineering, vol. 15, no. 10, pp. 3367-3375, 2005.

[21] X. Wang and M. Wang, "Hyperchaotic Lorenz system," Acta Physica Sinica, vol. 56, no. 9, pp. 5136-5141, 2007. 


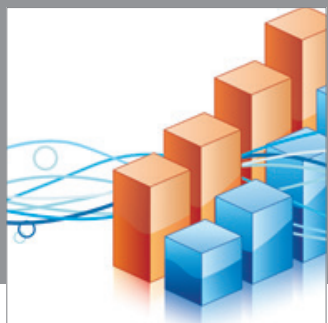

Advances in

Operations Research

mansans

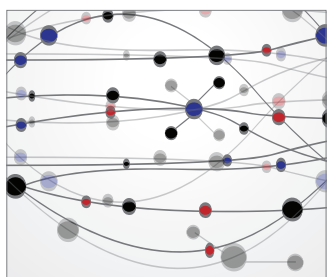

The Scientific World Journal
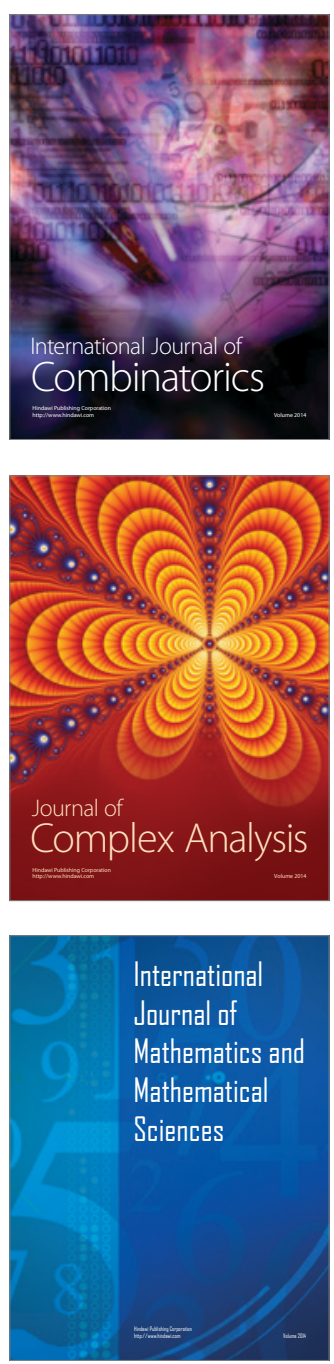
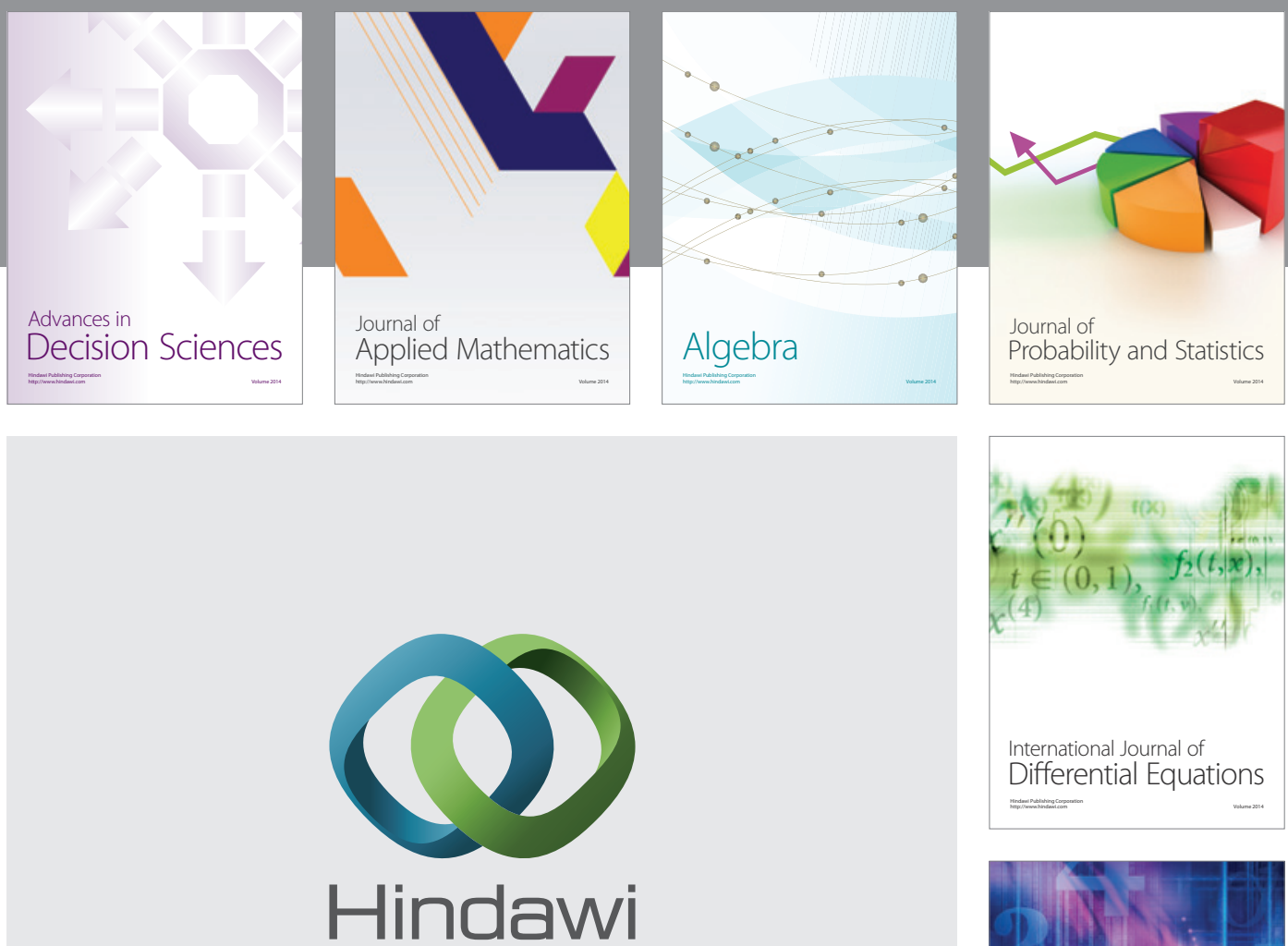

Submit your manuscripts at http://www.hindawi.com
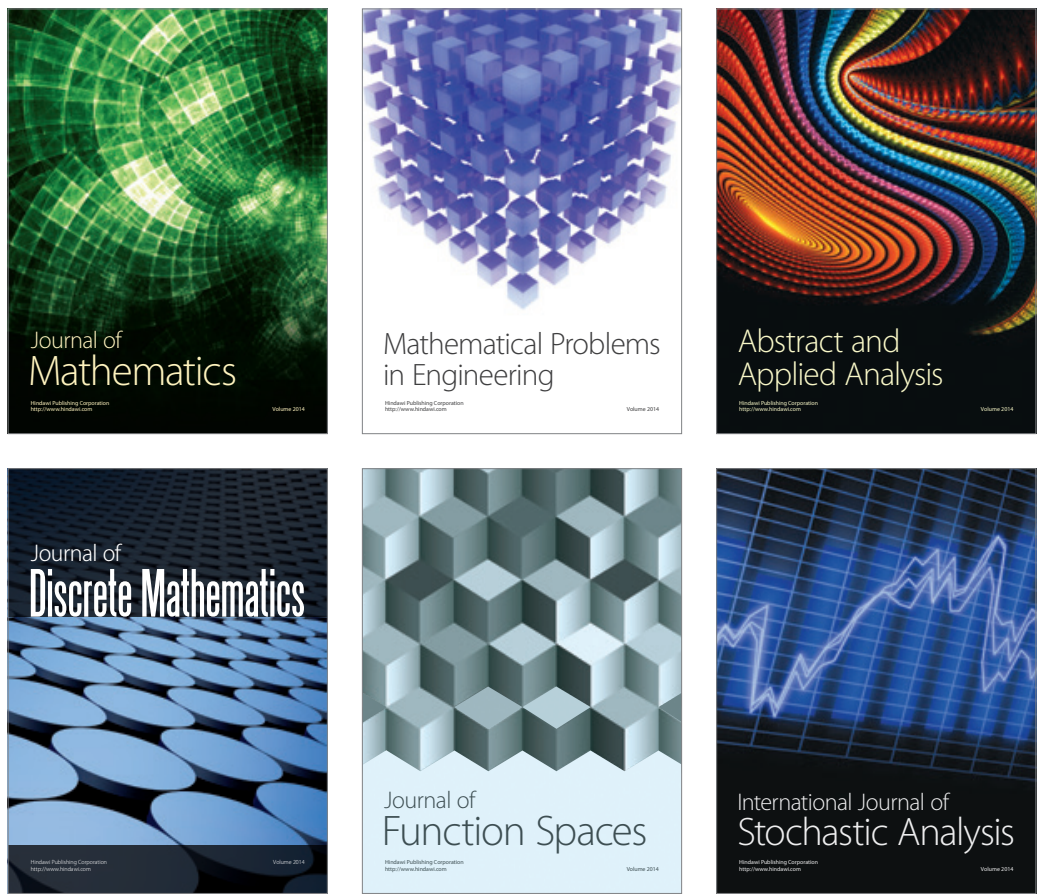

Journal of

Function Spaces

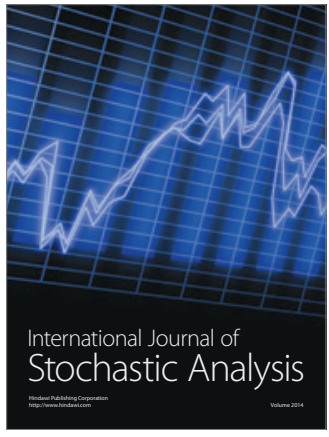

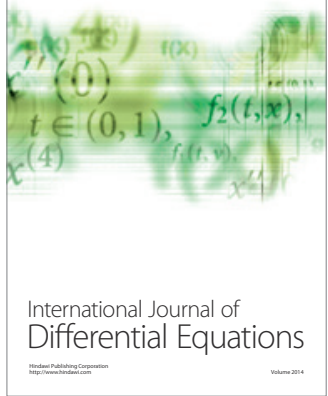
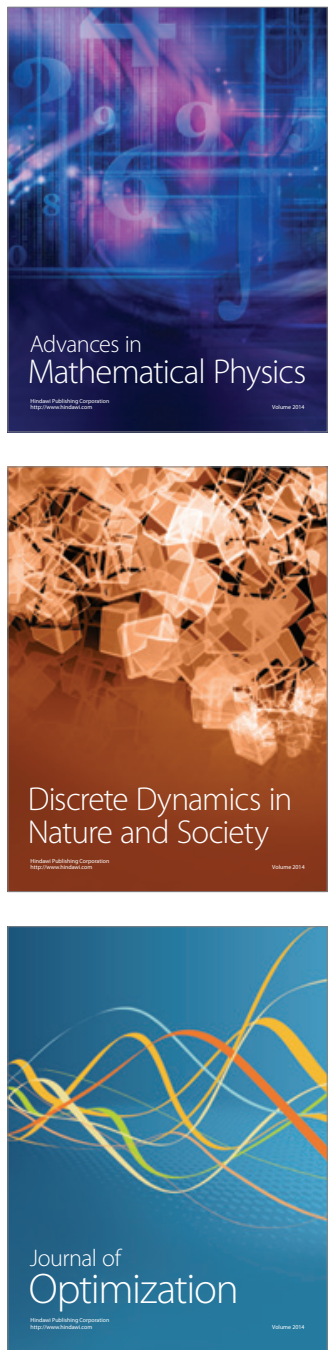